\title{
Críticas a las políticas de rendición de cuentas según lo que el profesorado chileno de secundaria considera justo en educación
}

\author{
Criticisms of accountability policies according to what Chilean secondary \\ school teachers consider «just» or «right» in education
}

Rocío Ferrada Hurtado'

\section{Resumen}

A partir del modelo De la justificación propuesto por Boltanski y Thévenot nos preguntamos cuáles son los bienes educativos que los profesores emplean para justificar lo que estiman justo hacer en la escuela, o para criticar una realidad escolar alejada de dichos ideales. Realizamos16 focus group donde participaron en total 68 docentes de colegios públicos, con financimiento mixto, y privados, de la ciudad de Santiago. Encontramos que identifican una situación escolar justa cuando ella refiere a una pluralidad de bienes educativos: de éstos, uno esencial es la preocupación por la individualidad del estudiante y la relación afectivo-personal profesor-alumno. Desde esta posición, las políticas de rendición de cuentas diseñadas en nombre de la estandarización de las prácticas y de la identificación de los aprendizajes a la obtención de resultados, son vistas como poco representativas de su trabajo por no respetar la diversidad ni las necesidades afectivo-personales de los estudiantes, y por identificar los fines escolares solo con la obtención de resultados eficientes en algunas disciplinas. En algunos casos, además, ésta los fuerza a ajustar sus prácticas a las metas comprometidas, llevándolos en ocasiones a renunciar a otros bienes educativos que valoran, o a conservar su adhesión a lo que consideran justo, pero a condición de distanciarse todo lo (poco) que les es posible, de los objetivos que persigue la autoridad educativa.

\section{Palabras clave}

Crítica docente, principios de justicia, políticas de rendición de cuentas, justificación, Chile.

\section{Abstract}

Basing our analysis upon Boltanski's and Thévenot's proposed paradigm in De la justification, we first question the educational goods that teachers are using in order to justify what they consider to be just in a school setting, or what seems open to criticism when reality strays too much from what an ideal school should be. We have organised 16 focus groups comprising 68 teachers in the secondary school system of the city of Santiago, privately or publicly funded or both. We found that teachers identify a school situation as "just" or "right" when it refers to a multiplicity of educational goods, among which should primarily be noted a concern for the individuality of students and for the affective and relational link between teacher and student. Since this position, evaluation policies based upon the standardisation of practices and the identifying of learning strategies aiming at obtaining specific results have been criticised, owing to the fact that they do not respect the diversity of students nor their affective and relational needs, and they only seek to equate the aims of schooling with the ability to obtain specific results. We conclude by saying that evaluation policies are generally not considered by teachers to be representative of their work. Besides, such policies sometimes force them to conform their practice to previously set goals, thus forcing them to give up on other educational goods although they value them, or to continue in agreement to what they believe to be just or right and thus having to distance themselves, when possible, from the goals set by the educational authorities.

\section{Keywords}

Teaching criticism, principles of justice, accountability policies, justification, Chile 


\section{Introducción}

En el marco de las políticas de rendición de cuentas para regular el funcionamiento de la escuela y las prácticas docentes, una serie de investigaciones críticas con estas medidas han estudiado las formas cómo las y los profesores reaccionan frente a éstas, y el modo cómo ellas inciden en sus comprensiones respecto de su propio trabajo. Según el enfoque que adopten, en general, estas medidas subrayan dos tipos de efectos sobre las prácticas docentes: i) la desprofesionalización del trabajo docente o incluso su proletarización (Lawn y Ozga, 1988). Pues, si la autonomía del trabajo es la prueba que define a una profesión (Champy y Israël, 2009; Schön, 1994; UNESCO, 1996), estas investigaciones concluyen que los docentes verían cuestionada -ellos mismos dudarían de- la efectiva realidad de su calificación en tanto profesionales; ii) los cambios en la comprensión de la identidad docente. Las nuevas maneras en que los docentes deben dar cuenta de su responsabilidad, se identifican (reducen) a ciertos objetivos mensurables y descontextualizados, soslayando o desestimando otros aspectos típicos y relevantes de su actividad (Comber y Nixon, 2009; Falabella, 2016; Ball, 2003).

Pero las regulaciones normativas a la institución escolar y a las prácticas de los actores educativos no son elementos extraños a estos sistemas; al contrario, ellas han sido una constante de su organización desde su fundación. Es, de hecho, el caso de los sistemas latinoamericanos (Toro, 2002), y en particular, del sistema educativo chileno (Núñez Prieto, 2004; Labarca, 1939; Toro, 2002). Entonces, en un contexto desde siempre regulado, las críticas docentes a las nuevas políticas no rechazarán tanto su existencia en cuanto tal, sino la reducción que éstas operan sobre lo que algunos han definido como la «irreductible complejidad» del trabajo docente, o el carácter plural que lo caracteriza (Ball, 2003; Derouet, 1992; Labaree, 2000).

El trabajo que aquí presentamos se inscribe en esta última crítica, pues notamos que las distintas investigaciones sobre las políticas de accountability no han sido suficientemente trabajadas a partir de la idea de pluralidad normativa que caracteriza, a nuestro juicio, las prácticas docentes. Apoyados en el modelo De la justificación desarrollado por Boltanski y Thévenot (1991), planteamos como hipótesis la existencia de una pluralidad normativa o de principios, presentes en las acciones de actores docentes. Sugiere el modelo que los actores, dotados de capacidades críticas, actúan teniendo en cuenta tanto la propia contingencia de la situación de su acción, como las distintas normas o principios que la regulan. Esta pluralidad de principios les permitiría desarrollar un ejercicio reflexivo y crítico de su acción, ya sea para justificar ante otros lo justo o adecuado de aquello que hacen, o para criticar lo injusto del orden en el que actúan, refiriendo sus justificaciones a dichos principios o referentes normativos.

En lo que sigue, presentamos, primero, algunos resultados de investigaciones internacionales y nacionales sobre la implementación de las políticas de accountability educativo y sus efectos sobre las prácticas docentes. Segundo, exponemos nuestra metodología de investigación que si bien, no tuvo como objetivo explícito conocer los efectos de dichas regulaciones, sino conocer los principios en los que las y los docentes apoyan sus justificaciones respecto de lo que consideran justo en educación, el tema de las políticas de rendición de cuentas apareció de todas formas y en reiteradas ocasiones. Presentamos aquí los resultados respecto a este punto, y algunas conclusiones a la luz de nuestra investigación y en diálogo con los estudios referidos. 


\section{Las políticas de accountability educativo en el contexto internacional}

A partir de la década de los 90, los sistemas escolares de distintos países del mundo se ven sometidos a las políticas de accountability o rendición de cuentas. Ellas corresponden a una serie de medidas tales como la aplicación de evaluaciones estandarizadas, la publicación de sus resultados y la incorporación de incentivos con miras a alcanzar determinados estándares y metas nacionales (Falabella y Opazo, 2014). Estas medidas, que suponen un actor racional estratégico que frente a incentivos, ajusta sus prácticas a las metas definidas, y se compromete con dichas medidas -pues serán ellos quienes las implementarán-, son propias del enfoque del modelo de mercado en educación y la Nueva Gestión Pública (New public management) actualmente dominantes en la organización de los sistemas educativos a nivel general (Hursh, 2005; Apple, 2007; Dupriez y Dumay, 2011; Joiko, 2012; Laval, et al., 2012; Amigot Leache y Martínez, 2015).

Este modelo concibe la educación como un bien adquirible en el mercado, pues asigna a éste un valor ante todo económico y de beneficio personal. Su postulado principal supone la competencia como factor de mejoramiento de la calidad de la educación. Para funcionar, el mercado requiere sin embargo de Estados comprometidos con el establecimiento de una serie de condiciones que aseguren su buen funcionamiento. Es decir, el mercado no se autorregula sino que precisa de la intervención gubernamental, que crea las condiciones para su existencia y desarrollo (Hursh, 2005; Sassen, 2009). Así, en educación, éste tendrá por tarea regular, controlar y evaluar el rendimiento del sistema a fin de producir información útil a los actores para que puedan realizar elecciones educativas informadas (bajo esta comprensión, identifica calidad de educación a estándares e indicadores mensurables, necesarios a la comparación del desempeño de los distintos establecimientos). Las políticas de accountability corresponden al tipo de dispositivos dirigidos a promover el funcionamiento del mercado - en efecto, la serie de supuestos que garantizan su funcionamiento, tales como la propia autoregulación o la sanción de las familias a los establecimientos con bajos resultados, no se observan en la realidad para el bien educativo (Dupriez y Dumay, 2011; Hursh, 2005)-, que los gobiernos aplican para «fortalecer la responsabilidad de los actores que deben dar cuenta del desempeño de su trabajo» (Raczynski y Muñoz, 2007: 40). Estas políticas fijan incentivos que orientan las prácticas de los actores; en general son de dos tipos: uno es la fijación, por parte del Estado, de estándares académicos mínimos que las escuelas deben alcanzar: según el nivel de logro de estos estándares, se las ordenará y se las premiará o sancionará. El otro, típico de los modelos de mercado-educativo, apuesta a la mejora de resultados mediante la elección escolar de los padres, que, en principio, escogerían la escuela que muestre los mejores resultados académicos y presionarían para que aquellas mantengan y/o aumenten sus resultados académicos (Dupriez y Dumay, 2011; Rouse y Barrow, 2008). Distintas evaluaciones de estas políticas a nivel internacional concluyen que aquéllas efectivamente inciden en las prácticas escolares; sus efectos pueden dar lugar a algunas mejoras del rendimiento, pero también a una serie de consecuencias, acaso no previstas. En el caso de los Estados Unidos, algunos estudios señalan entre sus virtudes conseguir mejorar en el rendimiento de las escuelas examinadas, o también orientar la acción educativa hacia objetivos claros y definidos (Puryear, 2006; West y Peterson, 2006). Otras reparan en cambio, sobre los efectos más perversos que ellas han generado en las prácticas educativas, tales como estrechar el currículo, privilegiando los contenidos que serán evaluados y aumentando la preparación de los exámenes (Jacob, 2005; Vazques Heilig y Darling-Hammond, 2008). A su turno, la revisión de Elacqua et al., (2013) sobre algunas de estas investigaciones, destacan que no solo los profesores reaccionan a las presiones del accountability distorsionando sus prácticas, sino también las autoridades locales y las/os directores de escuela. Otras evaluaciones notan que las modificaciones en las prácticas de los docentes son 
más recurrentes en los establecimientos donde las consecuencias del accountability son más drásticas (Pedulla et al., 2003) y otras destacan la existencia de prácticas derechamente indebidas que buscan trampear la aplicación de las evaluaciones y test (Jacob, 2005). También Coburn et al., (2016) analizan un conjunto de trabajos evaluadores de las políticas de rendición de cuentas en Estados Unidos, observando que el tipo de cambios en las prácticas pedagógicas que éstas denuncian dice más de un cambio «superficial», pues se aumentan las horas de enseñanza de los contenidos que serán evaluados y se ejercitan técnicas de logro, pero no se cambia la pedagogía ni las formas de aprendizaje de los estudiantes. Por su parte, en el caso de las escuelas inglesas, estudios de Day (2002) y Troman (2008) acentuaron sus efectos negativos sobre la identidad docente en su componente relativo a los factores afectivos en la enseñanza y el aprendizaje (citados en Ávalos, 2013).

Tenemos, entonces, que trabajos internacionales sobre los efectos de las políticas de accountability en las escuelas, evidencian transformaciones concretas en las prácticas pedagógicas de los actores educativos -cambios a veces superficiales (Coburn et al., 2016) y/o faltos de ética (Jacob, 2005)-, acotando de esta forma el sentido de la educación hacia logros académicos puntuales, estándares y mensurables. Por esa vía, dichas políticas identifican los fines de la educación y de la labor docente a lo que algunos han llamado una «cultura de la auditoría» (Apple, 2007), soslayando otros componentes de la educación y de las capacidades críticas de los actores docentes que intervienen.

\section{Las políticas de accountability en Chile}

Assaél et al., (2014) señalan que, tras una década de implementada la reforma educativa en Chile, cuyo propósito fue mejorar la calidad y equidad escolar ${ }^{2}$, el balance resultaba negativo si se tomaba como indicador los resultados de aprendizajes medidos por las pruebas estandarizadas nacionales (SIMCE) ${ }^{3}$ o internacionales (PISA, TIMSS). En ese contexto, sostienen los autores, se argumenta que no basta con entregar, como se había hecho hasta entonces, apoyo técnico y de recursos a las escuelas con bajos resultados académicos, sino que era preciso incrementar la presión - castigar a aquellos que lo «hacían mal» (Assaél et al., 2014)-, haciendo responsables a los actores educativos de sus desempeños y/o activos en el control del uso de sus dineros invertidos en educación (Corvalán y McMeekin, 2006; Raczynski y Muñoz, 2007). Se implementa entonces, en 2008, la Ley de Subvención Escolar Preferencial (SEP) que introduce por primera vez mecanismos explícitos de accountability tales como la clasificación de escuelas, y sanciones a aquellas que no cumplan con los estándares mínimos por desempeño. En breve, esta ley entrega recursos adicionales a las escuelas total o parcialmente subvencionadas por el Estado, que cuenten con estudiantes clasificados como vulnerables (prioritarios) y que de manera voluntaria quieran firmar un «Convenio de Igualdad de Oportunidades». Mediante este convenio, las escuelas se comprometen a mejorar sus resultados SIMCE, no seleccionar y retener a los alumnos, no cobrar arancel por alumnos prioritarios y a implementar un «Plan de Mejoramiento Educativo» (PME) definido por el mismo establecimiento ${ }^{4}$. La ley clasifica como «autónomas» a las escuelas que obtienen consistentemente resultados educativos buenos; «emergentes» a aquellas cuyos resultados varían, y «en recuperación» a las que obtienen malos resultados de manera regular: estas últimas arriesgan su cierre en caso de no alcanzar, durante tres periodos consecutivos, los estándares mínimos comprometidos.

\footnotetext{
2 Para conocer en detalle la serie de políticas implementadas en el marco de la reforma educativa en Chile ver García-Huidobro (1999), Cox (2003), Hevia (2003).

3 El SIMCE es el Sistema Nacional de Evaluación de resultados de aprendizaje del Ministerio de Educación. Este examen se aplica anualmente a distintos niveles escolares de primaria y al $2^{\circ}$ nivel de secundaria.

4 Para una explicación más en detalle del modo como funciona la ley SEP ver (Elacqua et al., 2013).
} 
Evaluaciones posteriores a su implementación (casos de estudio y etnografías), han arrojado resultados controversiales respecto de los supuestos y los beneficios esperados de la SEP. En el caso de las evaluaciones, una de ellas concluye mejoras en los aprendizajes de los estudiantes prioritarios de las escuelas de menores recursos, valoración por parte de los docentes del apoyo técnico pedagógico que reciben, focalización del trabajo en el mejoramiento de aprendizajes y en los resultados académicos (Raczynski et al., 2013) pero también un empeoramiento del clima escolar al concebirse a los alumnos prioritarios como un problema (Irarrázaval et al., 2012). La segunda evaluación, en cambio, de carácter cuantitativo, concluye que la mayor parte de las escuelas participantes no han logrado incrementar el rendimiento de los alumnos, ni tampoco es posible observar mejoras a nivel de la segregación socioeconómica del sistema (Valenzuela et al., 2012). Por su parte, al igual que algunos resultados internacionales, estudios nacionales encuentran que los docentes ajustan sus prácticas para aumentar los resultados en las pruebas estandarizadas, sin que ellos sean el reflejo de nuevas capacidades duraderas al interior de los establecimientos (Assaél et al., 2014; Falabella y Opazo, 2014; Elacqua et al., 2013; Falabella y de la Vega, 2016). Se trata pues de cambios superficiales e instrumentales, tales como la reducción curricular y/o el adiestramiento de los alumnos para responder pruebas estandarizadas. Es a la luz de resultados como estos que Falabella y Opazo (2014) concluyen que las políticas de Aseguramiento de la Calidad fomentan una racionalidad instrumental que prioriza el logro de metas y el cumplimiento de los procedimientos exigidos, trasladando a un segundo plano el análisis profesional autónomo y la toma de decisiones según criterios pedagógicos, contextuales y éticos. Advierten las autoras que ello podría incluso deteriorar los procesos de mejoramiento institucionales, al cuestionar la pertinencia de la gestión escolar de los establecimientos.

Asimismo, cuando los estudios se concentran en los efectos del accountability en los establecimientos calificados «en recuperación»-aquellos que obtienen permanentemente bajos resultados SIMCE- coinciden nuevamente con algunos trabajos internacionales en mostrar que los cambios descritos afectan aún más a estas escuelas. Así por ejemplo, Elacqua et al., (2013) observan que en ellas se reacciona aplicando medidas cortoplacistas ajustadas a aumentar los resultados, pero aún, que estas decisiones se toman sin la participación de los docentes, quienes en general -2 de cada 3 docentes- declaran no conocer la clasificación SEP de su establecimiento. Por su parte, Assaél et al. (2014) también acusan la falta de diálogo que se produce en estas escuelas, y la pérdida de sentido, producto del cuestionamiento a su existencia y/o su inminente desaparición. La realidad de estas escuelas, señalan, se ve amenazada al contrastarlas con un modelo ideal de escuela abstracto y descontextualizado, despojando así a los actores de su capacidad para abrir espacios de diálogo y construcción conjunta de los sentidos y el proyecto escolar que estimen. Finalmente, los efectos más perjudiciales en las escuelas «en recuperación» (mecanización del aprendizaje, postergación de los propios criterios profesionales en función de responder a los resultados estándares comprometidos) son también constatados por Falabella (2016), quien concluye que de esta forma, las políticas SEP tienden a legitimar y acrecentar la diferencia entre los establecimientos; en consecuencia, instiga a pensar en políticas más diferenciadas y flexibles, en acuerdo con las necesidades y características locales de los establecimientos.

De los resultados revisados nos interesa retener los efectos que la regulación heterónoma y estandarizada de la ley SEP tiene sobre las decisiones de los actores en situación: ésta no favorece la presencia de espacios de diálogo entre actores dotados de capacidades críticas y reflexivas para determinar aquello que es adecuado, conforme a la realidad escolar en la que actúan y en relación a las demandas de las políticas educativas. Por esta vía, dicha política trabaja sin considerar estas capacidades, ignorando los juicios pro- 
fesionales y éticos de los profesores sobre lo que creen justo hacer en sus contextos de trabajo. Veremos en lo que sigue, y a partir de nuestros resultados, cuáles son estas otras dimensiones del quehacer docente que quedan ignoradas y/o que los actores deben postergar, cuando, ante todo, se ha identificado el quehacer de la escuela al logro de resultados académicos estándares.

\section{Preguntas y metodología de investigación}

Advertimos más arriba que nuestra investigación no tuvo como objetivo explícito estudiar las políticas de accountabiliy y sus efectos sobre las prácticas de los docentes, sino que nos interesamos en conocer aquello que las y los profesores consideran que es justo hacer en sus contextos de trabajo, cuando se dan a la tarea de justificar sus prácticas ante otros (justificar por qué hacen lo que hacen, y justificar qué es lo que en realidad deberían hacer -o debería hacer la escuela-). Desde un enfoque de sociología pragmática como el desarrollado por Boltanski y Thévenot (1991) se postula que en las actuales sociedades, las personas o las instituciones refieren sus acciones de justificación a una pluralidad radical de bienes normativos o principios de justicia (Walzer, 1993; Boltanski y Thévenot, 1991; Derouet, 1992; Derouet, 2000). En este mundo plural, ningún principio se reduce o puede quedar contenido en otro; en consecuencia, los sujetos identificarán lo justo de una determinada situación (es decir, identificarán el bien normativo que importa dada la realidad de la situación) refiriendo dicha situación a principios de orden diferentes y opuestos entre sí. Los autores reconocen siete principios de justicia a partir de los cuales se establecen relaciones de orden entre los seres; estos modelos sirven a los actores para defender o criticar una determinada situación en caso que ella no se ajuste a su modelo normativo ideal. De estos siete regímenes generales de justicia, tenemos entre ellos el orden familiar o mundo doméstico que postula como bien común la autoridad, la lealtad, la confianza, pues concibe las relaciones entre los seres al modo familiar: esto es, marcadas por el afecto y la confianza entre los miembros, y adultos responsables del cuidado y la educación de los menores: pero se trata aquí de un tipo de relación jerárquica donde los adultos son responsables y modelos de comportamiento de los pequeños. Cuando los seres no cumplen con este orden (cuando un adulto no se ocupa de sus hijos o niños y/o cuando estos faltan a la autoridad del adulto) se critica la legitimidad de dicho ser, es decir, se pone en cuestión si acaso aquél es realmente lo que dice ser-si acaso el padre es realmente un padre-. Otro régimen de justicia es el orden o mundo industrial. Éste propone como bien común la eficiencia y los buenos resultados: en consecuencia, ordena a los seres según su aporte a la eficiencia de la empresa, cualquiera que ésta sea. En este mundo es central la planificación, el conocimiento científico-técnico, las mediciones, los resultados del trabajo. Otro, el orden o mundo cívico cuyo bien es la igualdad entre los seres (grupos, colectividades): aquí las relaciones estarán marcadas por la generalidad en el trato a los distintos grupos y la formación en saberes comunes y generales. Otro, el orden o mundo del mercado: su bien común es el dinero; establece relaciones de mercado entre los miembros, es decir, relaciones donde el bien de intercambio tiene un equivalente simbólico y es éste (el dinero) el que da acceso a entrar en dichas relaciones.

Así, en el «modelo del régimen de justificación», los actores, dotados como están de capacidades críticas, identificarán el bien que importa en una determinada situación (cívico, familiar, del conocimiento, el dinero,...), y en nombre de ese bien común justificarán sus críticas o adhesiones a la situación de su acción.

Desde dicho marco analítico, nos preguntamos qué órdenes normativos o principios de acción emplean los docentes para justificar sus acciones. Esta cuestión es importante, entre otras razones, por el hecho de que las decisiones de política educativa a menudo soslayan estos juicios, soslayan las capacidades reflexivas y los juicios éticos de los propios actores, tal como lo evidencian los resultados de las 
investigaciones sobre las políticas de accountability que hemos revisado. Realizamos entonces, en agosto de 2014, 16 focus group (grupos de discusión) donde participaron un total de 68 profesores/as de educación secundaria $a^{5}$ de todas las disciplinas, y de los tres tipos de establecimientos que componen el sistema educativo chileno: públicos subvencionados (4), particulares-pagados (5), y de subvención mixta (7) de la ciudad de Santiago de Chile ${ }^{6}$, todos contando con más de 5 años de experiencia docente. Del conjunto de establecimientos, 7 colegios fueron laicos y 9 de confesión católica ${ }^{7}$. La elección de la herramienta de recolección de los datos respondió a nuestro interés por conocer la forma que tomaba la reflexión de las y los docentes respecto de su propio trabajo, esto es, que los argumentos desarrollados avanzaran en generalidad a fin de justificarse en principios de justicia. El espacio que abre el focus gruop invita a las personas a entregarse a un trabajo reflexivo, pues, como ha explicado Giddens (1990) la reflexividad del propio quehacer se consigue en gran medida gracias al carácter conversacional del lenguaje, dado que la recusividad de éste le permite incluir en el proceso de construcción o producción de la reflexión, nuevas informaciones, representaciones, significados, opiniones de orden ético. A su turno, para conseguir argumentos fundados en principios morales, el focus gruop debía cumplir con dos condiciones: 1) la conversación debía ser pública (varios participantes) para que las personas construyesen relatos racionales y generales, y 2) las relaciones de fuerza entre las personas en presencia debían estar relativamente equilibradas, a fin que las personas se dieran libremente al disenso y la jerarquización de argumentos. De este modo los focus estuvieron compuestos por profesores y profesoras de secundaria, en grupos de 5 hasta 8 docentes, colegas de un mismo establecimiento, cuyas funciones eran principalmente pedagógicas (no incluimos ni directores/ as de establecimiento, ni jefes/as de ciclo). Concretamente, la conversación estuvo guiada por una pauta de entrevistas semiestructurada cuyos temas fueron la definición de su práctica de trabajo, que explicasen aquello que hacían y el por qué o para qué lo hacían, cuáles eran los límites de su trabajo, cuáles eran sus expectativas o ideales educativos y cómo confrontaban estos ideales con sus situaciones educativas reales. La principal estrategia adoptada para conocer la forma que tomaba la reflexión fue dejarlos avanzar muy libremente en la justificación de sus argumentos, y en ocasiones, empujarlos, mediante la pregunta «por qué» o «para qué», a avanzar hasta justificaciones morales de su acción.

La Tabla I presentada a continuación, contiene algunos elementos descriptivos de los establecimientos en los que realizamos los grupos de discusión y las siglas con las que, más abajo, identificaremos las citas seleccionadas.

\section{Tabla I. Descripción de las escuelas estudiadas}

\begin{tabular}{|c|c|c|c|c|}
\hline $\begin{array}{l}\text { DEPENDENCIA } \\
\text { ADMINISTRATIVA }\end{array}$ & $\begin{array}{c}\text { TAMAÑO } \\
\text { DE LA ESCUELA* }\end{array}$ & $\begin{array}{l}\text { ALUMNOS } \\
\text { POR CURSO }\end{array}$ & ORIENTACIÓN & $\begin{array}{c}\text { SIGLA } \\
\text { DE IDENTIFICACIÓN }\end{array}$ \\
\hline \multirow{4}{*}{ Liceo Público } & Mediano & 24 & Laica & $L P, M d, L$ \\
\hline & Mediano & 38 & Laica & $L P, M d, L$ \\
\hline & Mediano & 29 & Laica & $L P, M d, L$ \\
\hline & Grande & 38 & Laica & $L P, G r, L$ \\
\hline
\end{tabular}

5 En Chile existe solo un nivel de enseñanza secundaria obligatoria que dura 4 años, para las personas entre los 14 y 18 años. Después, ellas pueden o no continuar sus estudios de nivel terciario (universitario o técnico). Esta investigación ha sido realizada con profesorado del sistema formal.

6 A partir del año 2014 la ley SEP comienza a regir también para el nivel secundario. Según las cifras entregadas en 2014 por el Centro de Estudios del Ministerio de Educación, de un total de 11989 establecimientos municipales y particulares subvencionados que contaban con al menos un estudiante prioritario a nivel país, 7949 (66,3\%) establecimientos suscribieron un convenio SEP. De ellos, el 63\% eran establecimientos municipales y el $37 \%$ establecimientos particular-subvencionados.

7 No fue intención de la investigación trabajar con una muestra representativa de la realidad educativa chilena, ésta, al año 2014, se repartía de la manera siguiente: $38,4 \%$ del total de la matrícula de nivel primario y secundario, asistía a establecimientos públicos con subvención total, 52,3\% a establecimientos con subvención mixta y el 7,7\% a colegios particulares-pagados. 

según lo que el profesorado chileno de secundaria considera justo en educación

\begin{tabular}{|c|c|c|c|c|}
\hline $\begin{array}{l}\text { DEPENDENCIA } \\
\text { ADMINISTRATIVA }\end{array}$ & $\begin{array}{c}\text { TAMAÑ̃ } \\
\text { DE LA ESCUELA* }\end{array}$ & $\begin{array}{l}\text { ALUMNOS } \\
\text { POR CURSO }\end{array}$ & ORIENTACIÓN & $\begin{array}{c}\text { SIGLA } \\
\text { DE IDENTIFICACIÓN }\end{array}$ \\
\hline \multirow{7}{*}{ Colegio con Subvención Mixta } & Mediano & 36 & Católica & CSM,Md, $\mathrm{C}$ \\
\hline & Grande & 37 & Laica & CSM,Gr,L \\
\hline & Grande & 38 & Católica & CSM,Gr,C \\
\hline & Grande & 38 & Católica & CSM,Gr,C \\
\hline & Mediano & 36 & Laica & CSM,Md,L \\
\hline & Grande & 34 & Católica & CSM,Gr,C \\
\hline & Grande & 34 & Laica & CSM,Gr,L \\
\hline \multirow{5}{*}{ Colegio Particular Pagado } & Pequeño & 23 & Católica & $\mathrm{CPP}, \mathrm{Pq}, \mathrm{C}$ \\
\hline & Mediano & 24 & Católica & $\mathrm{CPP}, \mathrm{Md}, \mathrm{C}$ \\
\hline & Mediano & 25 & Católica & $\mathrm{CPP}, \mathrm{Md}, \mathrm{C}$ \\
\hline & Grande & 30 & Católica & $\mathrm{CPP}, \mathrm{Gr}, \mathrm{C}$ \\
\hline & Grande & 33 & Católica & $\mathrm{CPP}, \mathrm{Gr}, \mathrm{C}$ \\
\hline
\end{tabular}

*> 500 estudiantes: tamaño pequeño; Entre 500 y 1000: mediano; + de 1000 estudiantes: tamaño grande.

Fuente: Ministerio de Educación, 2014.

En estos espacios de reflexión, las y los profesores se refirieron inevitablemente a las políticas de control por resultado que han regulado el sistema educativo chileno desde la década de los 90, y que se han visto reforzadas a partir de la entrada en vigencia de la ley SEP en 2008. Del conjunto de información obtenida, destacaremos en esta exposición, i) otros bienes de la escuela o principios normativos -además del logro en los aprendizajes de los estudiantes priorizado por dichas políticas- que las y los docentes valoran como justos en educación, y a partir de los cuales apoyan sus críticas o adhesiones a las actuales medidas de accountability. Por juicio justo entendemos aquí un juicio realista, que tiene en cuenta los componentes de la situación, y ético, en el sentido que califica la situación en referencia a un bien común. ii) Las formas como los docentes, en sus situaciones cotidianas de aula, ajustan su labor a las distintas normativas de rendición de cuentas, develando en ese movimiento de ajuste el peso del orden normativo en toda su consistencia.

\section{Resultados}

Es consistente entre las y los profesores afirmar que la escuela debe responder, no únicamente a los aspectos puramente académicos e intelectuales de formación -como insisten las políticas de rendición de cuentas-, sino a múltiples fines, tales como la formación en conocimientos disciplinares, pero también la preocupación por cada uno de los estudiantes y su desarrollo, estimular su creatividad, su espíritu crítico, conseguir que todos, de igual manera, tengan acceso a los mismos saberes, la formación en las actuales tecnologías y redes sociales... Del conjunto de opiniones docentes destacaremos, por razones de espacio y pertinencia, solo uno de los regímenes de justicia referidos. Se trata del régimen familiar o «doméstico», que es el modelo que emerge con mayor fuerza, considerado por ellos/as como justo y eficaz para pensar las relaciones pedagógicas profesor-alumno.

\section{i. El modelo educativo familiar o «doméstico»}

Tanto los profesores de establecimientos subvencionados como privados, argumentan que es justo y clave en la escuela establecer y desarrollar relaciones afectivas y personales entre el profesor y sus estudiantes. Un modelo de relaciones de tipo familiar sirve a los docentes para apoyar sus juicios de justificación de aquello que hacen, o para criticar prácticas que consideran inapropiadas al trabajo docente. Este referente es movilizado para tres tipos de juicios: i) aquéllos que justifican las relaciones 
que tienen en cuenta la singularidad de los participantes, por considerarlas esenciales al trabajo docente, que es un trabajo con personas. En este caso el referente doméstico adquiere valor por sí mismo; ii) aquéllos donde se justifica el desarrollo de relaciones interesadas en la persona-estudiante, porque ellas son centrales para conseguir buenos aprendizajes. En este caso el principio doméstico se moviliza como complemento a otro referente, a saber, el del conocimiento o industrial, cuyo bien es el conocimiento disciplinar, ordenado y sistemático, sujeto a evaluación para medir la efectividad del aprendizaje; iii) aquéllos que ven en la práctica de relaciones de tipo familiar un modo no solo distinto, sino contrario al tipo de valoraciones estandarizadas y enfocadas al logro de los aprendizajes educativos promovidos por las actuales políticas educativas, y más ampliamente, contrarias al individualismo y las relaciones mercantilizadas propias de una sociedad neoliberal que acusan como dominante. En este caso, el mundo familiar o doméstico sirve de apoyo a los juicios críticos que denuncian la injusticia del actual orden escolar.

Todos los docentes justifican su quehacer en argumentos propios del mundo doméstico, pero aquellos que trabajan en colegios subvencionados (total o parcialmente) los refieren más frecuentemente; en los colegios particulares-pagados hay un balance en cambio, entre este tipo de argumentos y los argumentos que apelan a nociones académicas y de aprendizaje de saberes, más propias del mundo del conocimiento. Así, es en los liceos públicos donde se afirma con más regularidad como una práctica justa y necesaria al trabajo docente privilegiar los afectos y las relaciones interpersonales, incluso a veces sobre los aspectos académicos o disciplinares. Dicen «Y aqui nos preocupamos más de la niña que de los resultados. Si tiene problemas entonces nos ocupamos primero de eso y de los resultados es de lo último que nos preocupamos» (LP,Md,L). En otro grupo señalan: «es mucho más importante que el afecto esté bien en los alumnos que tanto énfasis en la parte académica. Un alumno que está bien va a aprenden»; o «el tema bumano es fundamental porque nosotros trabajamos con personas» (LP,Md,L). Por último, otros dicen: «ahora el profesor no es solo profesor sino que... inclusive es papá» (CSM,Gr,C). En estos colegios, las relaciones de tipo familiar se presentan como una suerte de trabajo necesario o condición de posibilidad para el trabajo netamente disciplinar y de aprendizaje de los estudiantes. Si esto es así, la política actual de accountability cometería un error al no considerar suficientemente este aspecto del trabajo pedagógico, pues los logros académicos que ellas buscan mejorar precisan de una dedicación igualmente importante en términos de las relaciones interpersonales profesor-alumno.

Ahora bien, los juicios docentes que priorizan las relaciones afectivas y de contención sobre otros bienes educativos, tienden a un tipo de práctica asimétrica en favor de un solo bien, que a una pluralidad de bienes para guiar la acción. Es posible que esta mirada asimétrica resulte de una exigencia realista y ética que prueban los profesores frente a estudiantes poco dotados de recursos afectivos. En efecto, para los profesores de liceos públicos, pero también para los que trabajan en establecimientos con subvención mixta, la carencia emocional y de contención familiar de sus estudiantes, adquiere un grado de realidad tal, que pide ser incorporada como un componente central del trabajo docente. El docente, señalan algunos profesores y profesoras de un colegio con subvención mixta, «termina resolviendo problemas sociales, personales... tiene que ver más con un proceso de socialización que con lo disciplinario que es en lo que nosotros debiésemos estar enfocados» (CSM,Gr,C). En sentido contrario, los profesores que enfrentan poblaciones mejor provistas de recursos domésticos, tienen mayores posibilidades de concentrarse en la enseñanza de su disciplina, sin necesariamente tener que detenerse demasiado en las demandas emocionales de sus estudiantes. 
Pero lo ideal de una práctica docente justa o apropiada es cuando ella refiere a una pluralidad simétrica de referentes, es decir, una co-participación de bienes educativos sin que uno prime sobre otro. Esta pluralidad es expresada por los docentes mediante lo que llamamos «nociones-puente», las cuales permiten juntar dos o más mundos a partir de los cuales justificar la acción. Así, por ejemplo, muchos profesores señalan que aquello que verdaderamente importa en la práctica pedagógica es «atender a la diversidad de los aprendizajes» o también el valor de los «progresos cotidianos del aprendizaje». Ambas nociones ponen en valor el bien del aprendizaje de los conocimientos, típico del mundo del conocimiento, al mismo tiempo que el bien de la diversidad de ritmos de cada estudiante, elemento afín al mundo doméstico. Las citas a este respecto son transversales y revelan hasta qué punto el trabajo docente enfrenta el desafío de aplicar métodos adecuados a la diversidad de los aprendizajes y, por tanto, desarrollar una práctica que debe tener en cuenta al menos dos bienes.

Dijimos asimismo que el principio familiar servía de apoyo a la justificación de juicios docentes críticos del actual orden escolar (punto iii). En general, los profesores reivindican este tipo de relaciones por ser no solo diferentes sino contrarias a los valores educativos incentivados por las políticas educativas, y contrarias también a la actual mercantilización de las relaciones sociales. Este modo de comprensión de lo doméstico es desarrollado casi por todos los colegios particular-subvencionados y por todos los colegios particulares-pagados. En particular, respecto de los juicios críticos de la política educativa, algunas citas dicen:

«Este colegio tiene la garantía de serprivado... con una personalidad propia, entonces el tema del SIMCE y el tema de la PSU [Prueba de Selección Universitaria] son pruebas que el colegio se preocupa de hacerlas, pero no es que estemos preparando a los niños durante todo un año para el SIMCE o la PSU» (CPP,Gr,C).

Y en otro establecimiento dicen: «los espacios chicos te generan mayores oportunidades en los vinculos... insisto, en este colegio hay otra mirada [contraria a la estandarización] ... tenemos un espacio privilegiado para romper esquemas» (CPP,Pq,C). Estos distintos ejemplos rescatan la distancia que el referente doméstico ofrece respecto de las políticas educativas que regulan el sistema educativo, distancia que los profesores de colegios privados califican como un privilegio social. Para estos docentes, las políticas generales de educación parecieran no operar como referentes generales de su acción docente sino, al contrario, serían vistas como medidas que, en el ideal, es preciso no seguir o en todo caso practicarlas mínimamente, intentando dejar espacio a prácticas de tipo familiar. Es elocuente la frase de un profesor de colegio grande con subvención mixta que dice:

«En cambio el colegio que se preocupa y tienes los planes y programas propios por lo menos tiene una intención, tiene una visión de lo que quiere entregar, en cambio un colegio que acepta lo que el Ministro o su equipo decida y lo aplica tal cual, claramente no está haciendo algo por cambiar las cosas...» (CSM,Gr,C).

\section{ii. Ajustes de la práctica docente a las normativas de rendición de cuentas}

Dado que las pruebas de evaluación de saberes han sido desde siempre el dispositivo por excelencia en educación, los profesores criticarán el SIMCE y otras evaluaciones, menos por su función que por el dominio que ejercen sobre la organización de los componentes escolares y las prácticas pedagógicas. La jerarquía y valor de los saberes escolares, la distribución de los tiempos, las decisiones pedagógicas, son organizadas en vista de lograr buenos resultados en las mediciones. Este ajuste es más denun- 
ciado por los docentes de los establecimientos subvencionados; los privados, en cambio, no refieren a este tema o solo lo hacen para describir el estado del sistema educativo en general, pero del cual ellos pueden en cierto modo sustraerse. De entre las citas de profesores que trabajan en establecimientos que han suscrito el convenio SEP, destacan:

«... porque de alguna manera los profesores que estamos en los focos SIMCE, nosotros trabajamos y presionamos y tensionamos a los alumnos y prácticamente los obligamos a muchas cosas: que tienes que venir hoy dia porque hay ensayo! Y todo nuestro trabajo del año hasta Noviembre es en base al SIMCE. Yo por estar en SIMCE tengo más horas porque tengo que generar material para planificar y todo» (LP,Md,L).

"Acá no está establecido [explícitamente] trabajar para el SIMCE pero si estamos igual sometidos a la presión del SIMCE, por dos aspectos: uno es porque hay un bono para los colegios que tienen buen SIMCE y dos, porque a los colegios que les va mal en el SIMCE pueden llegar a ser intervenidos e incluso cerrados» (CSM,Md,C).

«Todo ronda en torno al puntaje SIMCE, ensayo SIMCE, ensayo PSU. Al final la estandarización se convirtió en el fin» (CSM, Gr,C).

«Entonces genera una distorsión de la enseñanæa. Genera que los sistemas se tergiversen... Te premian dándote dinero por tres meses si tienes un SIMCE que esté por sobre el puntaje anterior; $Y$ ahora van a cerrar los colegios que obtienen mal puntaje... entonces todo se reduce a una fórmula y esa fórmula es la dimensión de un solo factor» (CSM,Gr,C).

«El sistema de por si empuja, casi obliga al profesor a evaluar, a ser competitivo, a rendir, nos evalúan por el SIMCE y la PSU...; Así como en la fábrica, mientras más artículos produzcan mejor, el alumno, mientras más rinda es mejor» (CSM,Gr,C).

Pero los profesores, al mismo tiempo que critican las medidas de control de sus prácticas, confirman que éstas pueden ser útiles e incluso justas al trabajo escolar. No hay solo crítica sino también confirmación o adhesión. En efecto, algunos profesores valoran una cierta normalización del trabajo o una intensificación de ciertos saberes evaluados por sobre otros, en nombre de la igualdad (referente cívico). Citas de profesores de colegios con subvención mixta sujetos al convenio SEP dicen: «Ahora también es cierto que con el bajo capital cultural que traen los chiquillos la normalización los ayuda» (CSM,Md,C). «Y en realidad los colegios como éste o como otros municipales, la única forma que tienen para tratar de equipararse con los otros es restando las horas de talleres y metiendo más horas de clasen» (CSM,Md,L); «Yo no encuentro que medir sea malo (...) el problema es que solamente está centrado abí) (CSM,Gr,L). A estas citas que destacan lo justo de las medidas de control por resultado y de las modificaciones pedagógicas que ellas consiguen, existen otras que incluso admiten un compromiso activo con mecanismos propios de este tipo de políticas como son los incentivos por resultados. Profesores de un colegio particular-subvencionado señalan: «Yo como sociedad le pedí que enseñara A: usted enseñó A y yo como sociedad lo premio. Y nosotros nos felicitamos entre nosotros cuando lo conseguimos. Lo criticamos ahora pero nos felicitamos si alguien lo consigue» (CSM,Gr,L).

La ambivalencia que prueban los docentes frente a las actuales regulaciones, es bien expresada por un profesor que dice «Es extraño porque todo parece ser que no queremos eso y a pesar de eso lo validamos y lo hacemos». Nos parece que en este ajuste de la acción docente a las obligaciones y normas de la situación, se 
revela todo el peso de la realidad, pero también y al mismo tiempo, la producción de la normatividad por parte de los propios actores. Tenemos de un lado el poder de las políticas de rendición de cuentas en curso para modelar la acción docente, pero de otro lado, la propia producción de los actores de la normatividad que los regula. Su participación a la producción de la normatividad es directa, en la medida que consideran justo un tipo de normalización de los aprendizajes docentes a fin de igualar los saberes dispares de los estudiantes; pero indirecta, en el sentido que su crítica contra las medidas vigentes permanece aún a escala individual, privada, contenta con modificar su situación puntual pero sin avanzar hacia categorías generales necesarias a la activación del potencial político de cambio que porta su crítica sobre el orden escolar. Dicen por ejemplo docentes de un establecimiento con financiamiento mixto:

«ser medidos, porque quiéralo o no, nos van a mediry abi entre medio uno tiene que contextualizar de acuerdo a la necesidad del alumno y abi uno trata de bacer lo que puede con el alumno, que uno sueña que puede hacer» (CSM,Md,L).

De esta forma, si el peso de la realidad y su apariencia de inmodificable no impiden el ejercicio de la crítica, el carácter privado de la misma la vuelve al corto plazo una acción inofensiva contra la realidad. La dificultad que los actores prueban para corregir los órdenes que califican de injustos se debe, como hemos visto, a la fuerza de los actuales dispositivos de control, pero también a la escasez de tiempo para desarrollar una reflexión crítica. Desde un enfoque de sociología pragmática de la acción, detener la acción para problematizar la realidad e intentar modificarla, requiere de tiempo. Regular el tiempo de los actores es, en este sentido, regular su realidad. La escasez de este recurso, acusada en general por todos los docentes de nuestra investigación, y constatada por muchas otras investigaciones (OCDE, 2013), conlleva a que las y los profesores no cuenten con tiempo para criticar y modificar dispositivos que consideran injustos al orden escolar ${ }^{8}$. De las citas tenemos:

«Y el rebelarte solo significa quedarte solo y por ende quizá hasta cesante, porque no puedes rebelarte solo, no puedes irte contra esta corriente ni tampoco puedes dejar de preparar a los alumnos para las mediciones, porque obviamente si yo no rindo con lo que me está pidiendo la sociedad... se culpa al profe» (CPP,Pq,C).

La escasez de tiempo dificultaría, entonces, el desarrollo de las capacidades críticas de los actores docentes, llevándolos a ajustarse a normas que acaso rechazan, o a conformarse con encontrar ciertos «espacios de libertad» donde hacer coincidir todo lo posible sus ideales con la realidad. Vimos más arriba que uno de estos «espacios de libertad» viene dado por el referente doméstico, que propone un modelo de relaciones escolares diferentes al de las relaciones de estandarización y énfasis en los resultados de las políticas de accountability. Este espacio se encarna en el aula. En lugar de seguir la norma, ciertos profesores se precian de la distancia que la sala de clases les ofrece para alejarse de aquella. Para ellos, es en el aula donde se da más nítidamente la relación profesor-alumno. En voz de un profesor: «ese es el espacio inherente a la profesión de docencia que es estar con el alumno» (CSM,Gr,L). Es en el aula donde el profesor prueba sus capacidades en tanto sujeto crítico y reflexivo capaz de ejercer una distancia entre la norma y su aplicación según la situación. Sin embargo, todavía en estos casos, la práctica docente crítica queda confinada a los límites de la sala de clases y a la acción individual de cada docente que así lo estime establece con sus estudiantes.

8 En nuestra investigación encontramos también otros elementos importantes que explicarían la incapacidad de los profesores para convertir sus juicios críticos en modificaciones reales de sus situaciones. No obstante, no nos es posible desarrollar estos elementos en el presente trabajo. 


\section{Conclusiones}

Basados en nuestros resultados y en los de las investigaciones nacionales e internacionales aquí revisadas, una primera conclusión se impone con fuerza: el peso de las políticas de rendición de cuentas sobre las prácticas pedagógicas. Ellas influyen en la forma como se organizan los saberes en las escuelas, la forma como se toman decisiones académicas, el bien educativo que se privilegia. Para el caso chileno, al igual que Elacqua et al., (2013), observamos que la cultura del accountability provoca estos cambios sobre todo en los establecimientos subvencionados, y en particular, en aquéllos que arriesgan sanciones más altas. Por su parte, estas políticas suponen un modelo escolar ideal más próximo a los colegios que atienden a poblaciones mejor dotadas de recursos económicos y educativos. En general, en nuestra muestra, estos colegios enfatizan la formación disciplinar y atienden al desempeño académico de sus estudiantes, pero en una relación simétrica con otros bienes educativos como el familiar. En este sentido, se trata de colegios donde se intenta conservar una pluralidad de bienes escolares como modelos guías de la acción pedagógica.

Coincidimos entonces con Falabella (2016) y Falabella y Opazo (2014) en que el diseño de políticas de accountability tales como la SEP contribuye a la práctica de una racionalidad puramente instrumental que prioriza el logro de metas y el cumplimiento de determinados procedimientos, a la vez que tiende a legitimar las actuales diferencias entre los establecimientos: esto es porque supone un ideal de modelo escolar cercano al que describen profesores de establecimientos que trabajan con poblaciones privilegiadas.

Dijimos que la práctica docente refiere a una pluralidad de bienes normativos, y dentro de estos, uno fundamental es el desarrollo de los aspectos personales y de la singularidad de los alumnos. Este bien es reivindicado por profesores de los distintos establecimientos, pero dicha reivindicación es aún más frecuente en las y los docentes de establecimientos subvencionados que trabajan con poblaciones acaso más demandantes de recursos afectivos. Sin embargo, dicho bien es soslayado por la política actual de accountability, centrada como está en el logro de resultados académicos. De este modo, ella elude un bien no solo esencial al trabajo pedagógico y colaborador en la tarea de aprendizaje de los estudiantes, sino también un aspecto dominante de la realidad de los establecimientos subvencionados a los que la SEP se dirige.

El desajuste que las y los docentes prueban entre la norma educativa y la realidad escolar que enfrentan, abre el espacio para la crítica, incluso si los mecanismos de control sobre sus prácticas y la falta de tiempo que a menudo acusan, atentan contra el desarrollo de un ejercicio crítico que modifique aquello que reclaman como injusto. Ball (2015) ha visto en estas acciones el germen de un cambio. Sostiene el autor que en contextos de gobernabilidad neoliberal, la subjetividad de los actores se revela como un lugar clave de lucha política. Siguiendo a autores como Foucault o Deleuze, Ball muestra que el espacio de reflexión de los docentes frente a ciertas normativas, opera como un ámbito no solo de libertad para imaginar distintas posibilidades de acción, sino de rechazo activo frente a ellas. Concibe entonces la subjetividad como el punto de partida para una política del rechazo (politics of refusal). Compartimos con Ball que el rechazo es necesario a una política que organice la escuela a partir de nuevas relaciones, pero todavía insuficiente para generar un cambio efectivo. Una política de cambio escolar necesitará de la reunión de actores docentes que salgan del aula, que dejen lo privado, e identifiquen su actual crítica (su rechą̧o en los términos de Ball) a nociones generales y compartidas con otros/as docentes de lo que creen justo en educación.

En fin, tenemos que las y los docentes critican las políticas generales de educación por no considerarlas representativas de los bienes que estiman justos: a saber, el aprendizaje, pero también el desarrollo personal de los estudiantes, o el desarrollo de su espíritu crítico, la capacidad de trabajar en colaboración y no en 
competencia,... Contrario a dicha pluralidad, las políticas chilenas de Aseguramiento de la calidad educativa que, en el ideal, debieran operar como referentes generales de la acción docente y que requieren de docentes comprometidos con su aplicación, son vistas en cambio como representativas de la estandarización de las prácticas, la reducción de la enseñanza a saberes cuantificables y comparables. Ellas pueden llevar a las y los profesores a enfrentar tensiones como i) renunciar a prácticas pedagógicas que consideran justas a sus realidades - que consideran realistas y éticamente adecuadas a su trabajo- concentrándose en la aplicación de los procedimientos actuales de Aseguramiento de la calidad. En estos casos, se arriesgaría, por ejemplo, la proletarización del trabajo docente en el sentido descrito más arriba por Lawn y Ozga (1988). O al contrario, ii) valorar como acción pedagógica justa aquella que no sigue la política educativa diseñada por las autoridades, distanciándose de ellas y simplemente trabajando todo lo (poco) que les sea posible, de manera distinta a los fines que las actuales políticas educativas buscan. En cualquiera de estos casos -y como también lo advierte Assaél et al., (2014) - las capacidades críticas de las y los docentes se ven afectadas al reducir el espacio para la reflexión, el diálogo y su compromiso con medidas que consideren lo que consideran justo hacer en sus contextos pedagógicos.

\section{Referencias Bibliográficas}

Amigot Leache, Patricia y Martínez, Laureano (2015): "Procesos de subjetivación en el contexto neoliberal. El caso de la evaluación del profesorado y la investigación universitaria". Revista de la Asociación de Sociología de la Educación, 8 (2), 138-155.

Apple, Michael (2007): "Education, Markets, and an Audit Culture". International Journal of Educational Policies, 1(1), 4-19.

Assaél, Jenny; Acuña, Felipe; Contreras, Paulina y Corbalán, Francisca (2014): “Transformaciones en la cultura escolar en el marco de la implementación de políticas de accountability en Chile. Un estudio etnográfico en dos escuelas clasificadas en recuperación”. Estudios Pedagógicos, XL (2), 7-26.

Ávalos, Beatrice (2012): “Cómo ven su identidad los docentes chilenos”. Perspectiva Educacional, 51 (1), 57-86.

Ávalos, Beatrice (2013). ¿Héroes o villanos? La profesión docente en Chile. Santiago: Universitaria.

Ball, Stephen J. (2003): The teacher's soul and the terrors of performativity. Education policy, 18(2), 215-228.

Ball, Stephen J. (2015): “Subjectivity as a site of struggle: refusing neoliberalism?” British Journal of Sociology of Education, 37 (8), 1129-1146. doi:10.1080/01425692.2015.1044072

Boltanski, Luc (1990). L'amour et la justice comme compétences. Trois essais de sociologie de l'action. Paris: Editions Métailié.

Boltanski, Luc y Thévenot, Laurente (1991). De la justification: les économies de la grandeur. Paris: Gallimard.

Bos, María Soledad Ganimian, Alejandro J., y Vegas, Emiliana (2014). América Latina en PIS A 2012. Brief 11: Chile en PIS A 2012. Logros y desafíos pendientes. BID, OCDE.

Calvo, Gloria (2006): "La pregunta por la enseñaza y el aprendizaje en el oficio docente” en E. Tenti (ed.), El oficio docente: vocación, trabajo y profesión en el siglo XXI. Buenos Aires: Siglo XXI, Argentina.

Champy, Florent y Israël, Liora (2009): "Professions et Engagement Public". Sociétés Contemporaines(73), 7-19. doi:10.3917/soco.073.0007 
Coburn, Cynthia E.; Hill, Heather C.; Spillane, James P. (2016): "Alignment and Accountability in Policy Design and Implementation: The Common Core State Standards and Implementation Research". Educational Researcher, 45 (4), 243-251. doi:10.3102/0013189X16651080

Comber, Barbara y Nixon, Helen (2009): “Teachers' work and pedagogy in an era of accountability”. Discourse: Studies in the Cultural Politics of Education, 30 (3), 333-345.

Corvalán, Javier y McMeekin, Robert (2006). Accountability educacional: posibilidades y desafíos para América Latina a partir de la experiencia internacional. Santiago: CIDE-PREAL.

Cox, Cristián (2003): "Las políticas educacionales de Chile en las últimas dos décadas del siglo XX" en C. Cox (ed.), Políticas educacionales en el cambio de siglo. La reforma del sistema escolar de Chile. Santiago: Universitaria.

Derouet, Jean-Louis (1992). École et justice: de l'égalité des chances aux compromis locaux. Paris: Métailié.

Derouet, Jean-Louis (2000). L'école dans plusieurs mondes. Bruxelles: De Boeck.

Dewey, John (1995). Democracia y Educación. Madrid: Morata.

Dupriez, Vincent y Dumay, Xavier (2011): “Les quasi-marchés scolaires : au bénéfice de qui?”. Revue française de pédagogie, 176, 83-100. doi:10.4000/rfp.3201

Elacqua, Gregory, Martínez, Matías, Santos, Humberto, y Urbina, Daniela (2013): “Escuelas bajo amenaza: Efectos de corto plazo de las presiones de accountability de la Ley SEP en las políticas y prácticas docentes". Documentos de trabajo, 16.

Falabella, Alejandra (2016): “¿Qué aseguran las políticas de aseguramiento de la calidad? Un estudio de casos en distintos contextos escolares”. Estudios Pedagógicos, XLII (1), 107-126.

Falabella, Alejandra y de la Vega, Luis Felipe (2016): "Políticas de responsabilización por desempeño escolar: Un debate a partir de la literatura internacional y el caso chileno”. Estudios Pedagógicos, XLII (2), 395-413.

Falabella, Alejandra y Opazo, Catalina (2014). Sistema de Aseguramiento de la Calidady procesos de mejora: Una mirada desde la gestión educativa. Informe ejecutivo. Santiago: Centro de Investigación de Desarrollo Educacional (CIDE), Universidad Alberto Hurtado

García-Huidobro, Juan Eduardo (1999). La reforma educacional en Chile. Santiago: Edición Popular.

Giddens, Anthony (1990). The consequences of modernity. Stanford: Stanford university press.

Hevia, Renato (2003). La educación en Chile boy. Santiago: Diego Portales.

Hursh, David (2005): "Neo-liberalism, Markets and Accountability: transforming education and undermining democracy in the United States and England". Policy Futures in Education, 3 (1), 3-15.

Irarrázaval, Ignacio; Paredes, R.; Murray, M.; Gutiérrez, G.; Bogolasky, F. y Contreras, C. (2012). Evaluación de los primeros años de implementación del Programa de Subvención Escolar Preferencial de la Subsecretaría de Educación. Informe de Investigación. Santiago: Centro de Políticas Públicas, Pontificia Universidad Católica de Chile.

Jacob, Brian A. (2005): "Accountability, incentives and behavior: The impact of high-stakes testing in the Chicago Public Schools”. Journal of Public Economics, 89, 761-796. 
Joiko, Sara (2012): "El cuasi-mercado educativo en Chile: desarrollo y consecuencias". Diálogos Educativos, $12(23), 148-174$.

Labarca, Amanda (1939). Historia de la enseñanza en Chile. Santiago de Chile: Imprenta Universitaria.

Labaree, David F. (2000): "On the Nature of Teaching and Teacher Education: Difficult Practices that Look Easy". Journal of Teacher Education, 51 (3), 228-233.

Laval, Christian; Vergne, Francis; Clément, Pierra y Dreux, Guy (2012). La nouvelle école capitaliste. Paris: La Découverte.

Lawn, Martin, y Ozga, Jenny (1988). “¿Trabajador de la enseñanza? Nueva valoración de los profesores”. Revista de Educación, 285, 191-215.

Ministerio de Educación. (4 de Agosto de 2014). Ficha Establecimiento. Obtenido de http://www.mime. mineduc.cl/mime-web/mvc/mime/portada

Núñez Prieto, Iván (2004). "La identidad de los docentes. Una mirada histórica en Chile”. Programa Interdisciplinario en Educación, PIIE, 1-18.

OCDE (2013). Education at a Glance 2013: OCDE Indicators. OECD Publishing (en línia) http:// dx.doi.org/10.1787/eag-2013-en, consultado el 09 de julio de 2017

Pardo, Marcela (2013). La visión de los docentes sobre las reformas educacionales en B. Ávalos, Héroes o villanos? La profesión docente en Chile. Santiago: Universitaria.

Pedulla, Joseph J.; Abrams, Lisa M.; Madaus, George F.; Russell, Michael K.; Ramos, Miguel A.; Miao, Jing (2003). Perceived Effects of State-Mandated Testing Programs on Teaching and Learning: Findings from a National Survey of Teachers. Boston: Boston College.

Puryear, Jeffrey M. (2006): “La accountability en la educación: ¿Qué hemos aprendido?” en J. Corvalán, y R. W. McMeekin (edits.): Accountability educacional: posibilidades y desafíos para América Latina a partir de la experiencia internacional. Santiago: PREAL-CIDE.

Raczynski, Dagmar y Muñoz, Gonzalo (2007): "Reforma educacional chilena: El difícil equilibrio entre la macro y micropolítica”. Serie estudios socio/ económicos, 31, 1-78.

Raczynski, Dagmar; Muñoz, Gonzalo; Weinstein, José y Pascual, Javier (2013): “Subvención Escolar Preferencial (SEP) en Chile: un intento por equilibrar la macro y micro política escolar. REICE”. Revista Iberoamericana sobre Calidad, Eficacia y Cambio en Educación, 11 (2), 164-193.

Rouse, Cecilia Elena, y Barrow, Lisa (2008): "School Vouchers and Student Achievement: Recent Evidence, Remaining questions". Annual Review of Economics, 1, 17-42.

Sassen, Saskia (2009). Critique de l'Etat. Territoire, Autorité et Droits, de l'époque médievale à nos jours. Paris: Demopolis/Le monde diplomatique.

Schön, Donald (1994). Le Praticien réflexif . À la recherche du savoir caché dans l'agir. Québec:les Éditions logiques.

Toro, Pablo (2002): "Nuevos recuerdos de las viejas escuelas: notas sobre la historia de la educación escolar en Chile y algunos de sus temas emergentes”. Persona y sociedad, XVI (2), 125-140. 
UNESCO. (1996). Recomendaciones de la $45^{a}$ Conferencia Internacional de Educación de la UNESCO. Ginebra: Oficina Internacional de Educación.

Valenzuela, Juan Pablo; Villarroel, Gabriel y Villalobos, Cristobal (2012). SEP: Algunos resultados preliminares en su implementación. Santiago: Borrador CIAE-Universidad de Chile.

Vazques Heilig, Julian y Darling-Hammond, Lisa (2008): “Accountability Texasstyle: The progress and learning of urban minority students in a high-stakes testing context". Educational Evaluation and Policy Analysis, 30 (2), 75-110. doi:10.3102/0162373708317689

Walzer, Michael (1993). Las esferas de Justicia. Madrid: Fondo de Cultura Económica de España.

West, Martin R., y Peterson, Paul E. (2006): WThe efficacy of choice threats within school accountability systems: Results from legislatively induced experiments". The Economic Journal, 116 (510), C46-C62.

\section{Nota biográfica}

Rocío Ferrada Hurtado es socióloga por la Universidad Alberto Hurtado de Chile; Máster en Gestión y Políticas Públicas por la Universidad de Chile; Doctora en Ciencias Políticas y Sociales por la Université Catholique de Louvain y, en la actualidad, Becaria del Programa Becas Chile, CONICYT. 